Letter to the Editor

\title{
How the loss in academics and research occurs by stopping animal experimentation in medical curriculum
}

\author{
Garima Adhaulia*, Shireen Barua, Divya Singh, Suchi Jain, Sayed Shakita Fatima, \\ Sanjay Kumar, Amod K. Sachan, Rakesh K. Dixit
}

Sir,

Use of animals in experimentation and research has always been a topic of great debate. Some express their strong support while others are against animal research practices and want their complete abolition. ${ }^{1}$ At present, there is a pill for every ill. Rapid advancement in the field of science and technology contributed in discovering cure and medications even for the rarest of the rare diseases. Most of the present day discoveries in medical science lay their foundation on animal experimentation. The use of drugs in clinical practice have been possible only after going through successful animal studies for safety, efficacy and toxicity.

Animal contribution towards medical science ranges from detailed understanding of basic human physiology to drug discovery. ${ }^{2}$ Example of some of the revolutionary drug and vaccine discoveries are - production of diphtheria antitoxin and the development of a vaccine against diphtheria and tetanus by Behring using horses lead to the first Noble prize in physiology or medicine in 1901, Insulin was first isolated from dogs in 1922, antibacterial activity of prontosil was demonstrated by Domagk in 1939 by experiments on chicken, antibiotic treatment and vaccines for leprosy were developed using armadillos in 1970s. Steroid administration in premature lambs and rabbits 30 years ago, led to lung development which in turn influenced the survival rates of premature babies. ${ }^{3,4}$

Animal model of diseases helps us to understand the exact pathophysiology of the diseases and to screen the potential treatment and cure for the same. Example - Mouse models for heart condition in Marfan's syndrome resulted in potential new treatment. Mouse and sheep models of cystic fibrosis led to improvements in gene therapies. Immunization against human papilloma virus (HPV) was developed following research in rabbits, dogs and cattle showing cervical cancer can be stopped by vaccination. ${ }^{4}$ Animal research has facilitated the study of neurobiology of various psychiatric disorders and receptors of various drugs used in human subjects. ${ }^{5}$

Usage of animals in medical education and research is of paramount significance. For the past many years, animal experimental sessions formed the base of teaching medical subjects especially pharmacology, physiology and surgery. ${ }^{6}$ The basic objective of teaching is always to provide the detailed understanding of the subject rather than just mugging up the concepts without understanding. Functioning of biological system of animals show close resemblance with humans and more than $90 \%$ of the human genes have equivalents in mice and rats which helped us to better understand the complexity and physiology of human body through animal experiments. ${ }^{4}$ In teachings for undergraduate and postgraduate demonstration of mechanism of action of various drugs, bioassay-to determine the nature of unknown drugs, receptor discovery, screening of various drugs, learning of clinical skills e.g. administering drugs and preclinical testing including general and specific toxicity studies have been possible because of animal experimentation. ${ }^{3}$

Many animal rights protection groups criticize the use of animals in research and education as most of the laboratories and institutions are lacking in following the good laboratory practices and ethics. Extensive use of irrelevant procedures like cosmetic testing raised questions on animal use and its utility.

For this reason, various alternative methods such as computer simulation models, in vivo, in vitro techniques, audio visual techniques etc are initiated in teaching as well as in research. Though these alternative methods can simulate the picture but can't provide the better understanding and creation of life long memory.

Scientists and researchers across the world are always in favour of judicious use of animals in various experiments and researches. Alternative methods can be cost effective and easy to perform but still the importance of animals in research and development can't be denied completely. In order to safeguard the animal rights it is advisable to perform only those procedures under standard laboratory conditions on animals that are extremely beneficial to the mankind in future. Immediate positive results may not be encountered in animal research but practicing it ethically can definitely bring wonders in the field of science and research.

Garima Adhaulia, Shireen Barua, Divya Singh, Suchi Jain, Sayed Shakita Fatima, Sanjay Kumar, Amod K. Sachan, Rakesh K. Dixit 
Department of Pharmacology and Therapeutics, K. G. M. U., Lucknow, Uttar Pradesh, India

*Correspondence to Dr. Garima Adhaulia, Email: drgarima.26@gmail.com

\section{REFERENCES}

1. Ormandy E H, Schuppli C A. Public Attitudes toward Animal Research: A Review. Animals (Basel). 2014;4(3):391-408.

2. Ressler KJ, Mayberg HS. Targeting abnormal neural circuits in mood and anxiety disorders: from the laboratory to the clinic. Nat Neurosci. 2007;10(9):1116-24.

3. Badyal D K, Desai C. Animal use in pharmacology education and research: The changing scenario. Indian J Pharmacol. 2014;46(3);257-65.
4. Understanding Animal Research. Animal Research: the facts-understanding animal research. Available at: http://www.understandinganimalresearch.org.uk/dow nload_file/1045/174. Accessed on 28 December 2018.

5. Nerlekar S, Karia S, Harshe D, Warkari R, Desousa A. Attitude and knowledge of undergraduate medical students towards the use of animals in medical research: an exploratory study. J Clin Diag Res. 2018;12(7):4-6.

6. Roy V, Tekur U. Animal experiments in medical undergraduate curriculum: A teacher student perspective. Indian J Pharmacol. 2001;33:104-7.

Cite this article as: Adhaulia G, Barua S, Singh D, Jain S, Fatima SS, Sanjay Kumar S, et al. How the loss in academics and research occurs by stopping animal experimentation in medical curriculum. Int $\mathbf{J}$ Basic Clin Pharmacol 2019;8:608-9. 